\title{
THE GENUS ZETA FUNCTION OF HEREDITARY ORDERS IN CENTRAL SIMPLE ALGEBRAS OVER GLOBAL FIELDS
}

\author{
M. DENERT
}

\begin{abstract}
Louis Solomon introduced the notion of a zeta function $\zeta_{\boldsymbol{\theta}}(s)$ of an order $\Theta$ in a finite-dimensional central simple $K$-algebra $A$, with $K$ a number field or its completion $K_{P}$ ( $P$ a non-Archimedean prime in $\left.K\right)$. In several papers, C. J. Bushnell and I. Reiner have developed the theory of zeta functions and they gave explicit formulae in some special cases. One important property of these zeta functions is the Euler product, which implies that in order to calculate $\zeta_{\boldsymbol{\Theta}}(s)$, it is sufficient to consider the zeta function of local orders $\Theta_{P}$. However, since these local orders $\Theta_{P}$ are in general not principal ideal domains, their zeta function is a finite sum of so-called 'partial zeta functions'. The most complicated term is the 'genus zeta function', $Z_{\boldsymbol{\theta}_{p}}(s)$, which is related to the free $\Theta_{p}$-ideals. I. Reiner and C. J. Bushnell calculated the genus zeta function for hereditary orders in quaternion algebras (i.e., $[A: K]=4$ ). The authors mention the general case but they remark that the calculations are cumbersome. In this paper we derive an explicit method to calculate the genus zeta function $Z_{\boldsymbol{\Theta}_{P}}(s)$ of any local hereditary order $\Theta_{P}$ in a central simple algebra over a local field. We obtain $Z_{\boldsymbol{\Theta}_{P}}(s)$ as a finite sum of explicit terms which can be calculated with a computer. We make some remarks on the programming of the formula and give a short list of examples. The genus zeta function of the minimal hereditary orders (corresponding to the partition $(1,1, \ldots, 1)$ of $n$ ) seems to have a surprising property. In all examples, the nominator of this zeta function is a generating function for the $q$-Eulerian polynomials. We conclude with some remarks on a conjectured identity.
\end{abstract}

Louis Solomon introduced, in [12], the notion of a zeta function $\zeta_{\Theta}(s)$ of an order $\Theta$ in a finite-dimensional central simple $K$-algebra $A$, with $K$ a number field or its completion $K_{P}(P$ a non-Archimedean prime in $K)$.

In several papers [1-5] C. J. Bushnell and I. Reiner have developed the theory of zeta functions and they gave explicit formulae in some special cases. Although the theory is formulated for number fields $K$ (or their completions $K_{P}$ ), it is immediately extended to global function fields $K$ (or their completions $K_{P}$ ).

One important property of these zeta functions is the Euler product, which states that for an $R$-order $\Theta$ in $A$, with $R$ a Dedekind ring in $K$, one has

$$
\zeta_{\boldsymbol{\Theta}}(s)=\prod_{P \text { prime in } R} \zeta_{\boldsymbol{\Theta}_{P}}(s) .
$$

Received May 7, 1987; revised April 15, 1988.

1980 Mathematics Subject Classification (1985 Revision). Primary 11S45, 16A18.

The author is supported by an N.F.W.O. grant. 
So, in order to calculate $\zeta_{\Theta}(s)$, it is sufficient to consider the local factors $\zeta_{\Theta_{P}}(s)$. However, the local $R_{P}$-orders $\Theta_{P}$ are in general not principal ideal domains, and their zeta function $\zeta_{\Theta_{P}}(s)$ is a finite sum of $h\left(\Theta_{P}\right)$ 'partial zeta functions', with $h\left(\Theta_{P}\right)$ the class number of the local order $\Theta_{P}$. One term is related to the free $\Theta_{P}$-ideals, the so-called 'genus zeta function' of $\Theta_{P}$, denoted by $Z_{\boldsymbol{\Theta}_{P}}(s)$.

In previous papers $[7,8]$ we studied the locally free class group of an $R$-order $\Theta$; therefore, our special interest concerns the genus zeta function of $\Theta$ :

$$
Z_{\boldsymbol{\Theta}}(s)=\prod_{P \text { prime in } R} Z_{\boldsymbol{\Theta}_{P}}(s)
$$

In [4] the factors $Z_{\Theta_{P}}(s)$ were calculated for hereditary orders in quaternion algebras (i.e., $[A: K]=4)$. (Actually, the authors of [4] determined $\zeta_{\Theta_{P}}(s)$ in this special case; the main difficulty, however, is the calculation of the genus zeta function.) The authors mention the general case but they remark that the calculations are cumbersome.

In this paper we derive an explicit method for calculating the local factors $Z_{\boldsymbol{\Theta}_{P}}(s)$ of the genus zeta function $Z_{\boldsymbol{\Theta}}(s)$ for any hereditary $R$-order $\Theta$ in a central simple algebra over a global field. The main idea is to use the description of the orbits in $\Theta_{P}$ under the left action of $\Theta_{P}^{*}$, cf. $\S 3$. This reduces the calculation to a counting problem, studied in $\S 2$. The necessary combinatorics needed to solve this problem are introduced in $\S 1$. We obtain $Z_{\Theta_{p}}(s)$ as a finite sum of explicit terms which can be calculated with a computer. We make some remarks on the programming of the formula and in the appendix we give a short list of examples, including all the partitions of $n \leq 5$.

With similar methods we calculated the partial zeta functions of hereditary orders. This allowed us to prove Solomon's second conjecture; cf. [1] for hereditary orders and also [10].

The examples of the genus zeta function of minimal hereditary orders (corresponding to the partition $(1,1, \ldots, 1)$ of $n)$ reveal a remarkable connection with the $q$-Eulerian polynomials. We conjecture that the nominator of this genus zeta function is a generating function for the $q$-Eulerian polynomials.

We verified this for $n \leq 20$; however, we do not have a proof of this relation for arbitrary $n$. In fact, we did not succeed in relating the inductive definition of the $q$-Eulerian polynomials to the constructive definition of the genus zeta function. In $\S 4$ we make some further remarks on this.

\section{The $\eta$-ADMissible PeRMUtations in $S_{n}$}

Let $S_{n}$ be the permutation group of $\{1, \ldots, n\}$. We can visualize $\sigma \in S_{n}$ by its graph $\Gamma_{\sigma}=\{(i, \sigma(i)) \mid 1 \leq i \leq n\}$ : we indicate the points of $\Gamma_{\sigma}$ in the lattice $\{1, \ldots, n\}^{2}$, see Figures 1 and 2 .

Since we will use the lattice to visualize the entries of matrices, cf. $\S 3$, the point $(i, j)$ is plotted, with $i$ vertical and $j$ horizontal. 


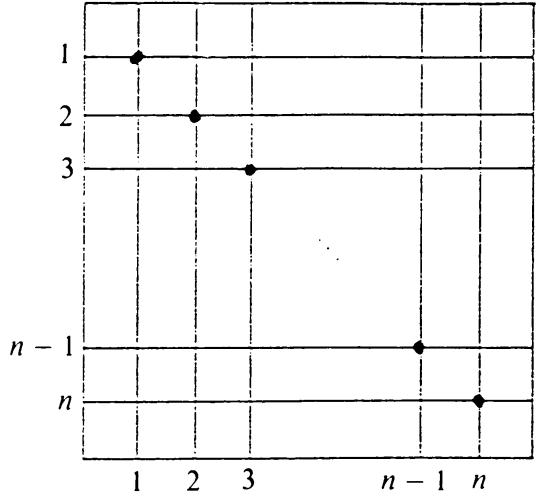

FIGURE 1. $\sigma=1_{n}$

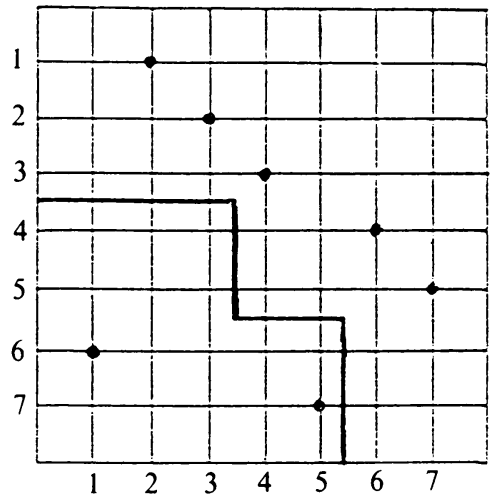

FIGURE 2. $\sigma=\left(\begin{array}{lll}1 & 2346)(57)\end{array}\right.$

Definition 1.1. For $I \subset\{1, \ldots, n\}^{2}$ we say that $\sigma$ is a descending line on $I$ if and only if for every $(i, \sigma(i)),\left(i^{\prime}, \sigma\left(i^{\prime}\right)\right) \in I \cap \Gamma_{\sigma}, i<i^{\prime}$ is equivalent to $\sigma(i)<\sigma\left(i^{\prime}\right)$.

Examples. (1) The identity $1_{n}$ is a descending line on every set $I$.

(2) $\sigma=(12346)(57)$ in Figure 2 is a descending line on $I=\{2,3,5\} \times$ $\{1, \ldots, 7\}$.

We now fix a composition $\eta=\left(n_{1}, \ldots, n_{r}\right) \in \mathbf{N}^{r}$, with $n_{i} \neq 0$ and $n=$ $\sum_{1 \leq i \leq r} n_{i}$. A composition $\eta$ will correspond to a partitioning of matrices into $r^{2}$ blocks of size $n_{i} \times n_{j}$, cf. Theorem 7 .

The $k$ th partial sum $(k \leq r)$ of $\eta$ is abbreviated by $S_{\eta}(k)=\sum_{1 \leq i \leq k} n_{i}$. We will frequently use the block-map $\eta^{*}$ associated with $\eta$ and defined by

$$
\eta^{*}:\{1, \ldots, n\} \rightarrow\{1, \ldots, r\} \text { such that } S_{\eta}\left(\eta^{*}(i)-1\right)<i \leq S_{\eta}\left(\eta^{*}(i)\right) .
$$

We say that the row $i$ belongs to the block $\eta^{*}(i)$. A block-row (resp. blockcolumn) is the subset of $\{1, \ldots, n\}^{2}$ containing $(i, j)$ with $\eta^{*}(i)\left(\operatorname{resp} . \eta^{*}(j)\right)$ constant. We also introduce the notation $i \prec j$ (resp. $i \succ j, i \approx j$ ) to indicate that $\eta^{*}(i)<\eta^{*}(j)$ (resp. $\left.\eta^{*}(i)>\eta^{*}(j), \eta^{*}(i)=\eta^{*}(j)\right)$.

Definition 1.2. A permutation $\sigma \in S_{n}$ is $(\eta)$-admissible if and only if $i \approx i^{\prime}$ and $i<i^{\prime}$ imply $\sigma(i)<\sigma\left(i^{\prime}\right)$. In other words, $\sigma$ is $\eta$-admissible if and only if $\sigma$ is a descending line on every block-row. The set of $\eta$-admissible permutations is denoted by $S^{(\eta)}$. Observe that $S^{(\eta)}$ is not a semigroup.

Example. $\sigma=(12346)(57)$ in Figure 2 is $(3,2,2)$-admissible but not $(2,2,3)$-admissible.

In view of the application to hereditary orders in Theorem 7 we separate the lattice $\{1, \ldots, n\}^{2}$ into two disjoint parts:

$$
\begin{aligned}
& {[i \preceq j]=\left\{(i, j) \text { with } \eta^{*}(i) \leq \eta^{*}(j)\right\},} \\
& {[i \succ j]=\left\{(i, j) \text { with } \eta^{*}(i)>\eta^{*}(j)\right\} .}
\end{aligned}
$$

We visualize this separation with a line as in Figure 2 for $\eta=(3,2,2)$. 
Definition 1.3. (i) The block numbers of $\sigma$ are

$$
b_{\sigma}(l, k)=\#\left\{(i, \sigma(i)) \mid \eta^{*}(i)=l \text { and } \eta^{*}(\sigma(i))=k\right\} \quad \text { for } 1 \leq l, k \leq r .
$$

(ii) The upper and lower row indices of $\sigma$ are

$$
\left\{\begin{array}{l}
r_{\sigma}^{+}(l)=\#\left\{(i, \sigma(i)) \mid \eta^{*}(i)=l \leq \eta^{*}(\sigma(i))\right\} \\
r_{\sigma}^{-}(l)=\#\left\{(i, \sigma(i)) \mid \eta^{*}(i)=l>\eta^{*}(\sigma(i))\right\}
\end{array} \quad \text { for } 1 \leq l \leq r .\right.
$$

(iii) The upper and lower column indices of $\sigma$ are

$$
\left\{\begin{array}{l}
c_{\sigma}^{+}(k)=\#\left\{(i, \sigma(i)) \mid \eta^{*}(i) \leq \eta^{*}(\sigma(i))=k\right\} \\
c_{\sigma}^{-}(k)=\#\left\{(i, \sigma(i)) \mid \eta^{*}(i)>\eta^{*}(\sigma(i))=k\right\}
\end{array} \quad \text { for } 1 \leq k \leq r .\right.
$$

We directly obtain the following

Properties. (1) For $1 \leq k<l \leq r$ we have

$$
\left\{\begin{array}{l}
r_{\sigma}^{+}(l)=\sum_{l \leq j \leq r} b_{\sigma}(l, j), \quad r_{\sigma}^{-}(l)=\sum_{1 \leq j<l} b_{\sigma}(l, j), \quad r_{\sigma}^{+}(l)+r_{\sigma}^{-}(l)=n_{l} ; \\
c_{\sigma}^{+}(k)=\sum_{1 \leq i \leq k} b_{\sigma}(i, k), \quad c_{\sigma}^{-}(k)=\sum_{k<i \leq r} b_{\sigma}(i, k), \quad c_{\sigma}^{+}(k)+c_{\sigma}^{-}(k)=n_{k} .
\end{array}\right.
$$

(2) For $1<l \leq r$ we have

$$
U_{\sigma}(l)=\sum_{1 \leq j<l} c_{\sigma}^{-}(j)-\sum_{2 \leq i<l} r_{\sigma}^{-}(i)=\sum_{l \leq i \leq r} r_{\sigma}^{-}(i)-\sum_{l \leq j<r} c_{\sigma}^{-}(j) \geq 0 .
$$

Proof. The first property is trivial, and for the second property we note that

$$
U_{\sigma}(l)=\sum_{l \leq i \leq r} \sum_{1 \leq j<l} b_{\sigma}(i, j) \geq 0 .
$$

Lemma 1. Consider a set of natural numbers $A=\{r(l), c(k) \mid 1 \leq k<l \leq r\}$ with $0 \leq r(l) \leq n_{l}$ and $0 \leq c(k) \leq n_{k}$ for $1 \leq k<l \leq r$, and denote

$$
S_{A}=\left\{\sigma \in S^{(\eta)} \mid r_{\sigma}^{-}(l)=r(l), c_{\sigma}^{-}(k)=c(k) \text { for } 1 \leq k<l \leq r\right\} .
$$

Then

(i) the set $S_{A}$ is nonempty if and only if for $1<l \leq r$

$$
\sum_{1 \leq j<l} c(j)-\sum_{2 \leq i<l} r(i)=\sum_{l \leq i \leq r} r(i)-\sum_{l \leq j<r} c(j) \geq 0
$$

(ii) in this case there exists a unique $\sigma_{d} \in S_{A}$ that satisfies the additional condition that $\sigma_{d}$ is a descending line on $[i \preceq j]$, on $[i \succ j]$, and on every block-column.

Proof. (i) It is clear that the conditions on $r(l)$ and $c(k)$ are necessary and sufficient conditions to obtain that $S_{A}$ is nonempty. 


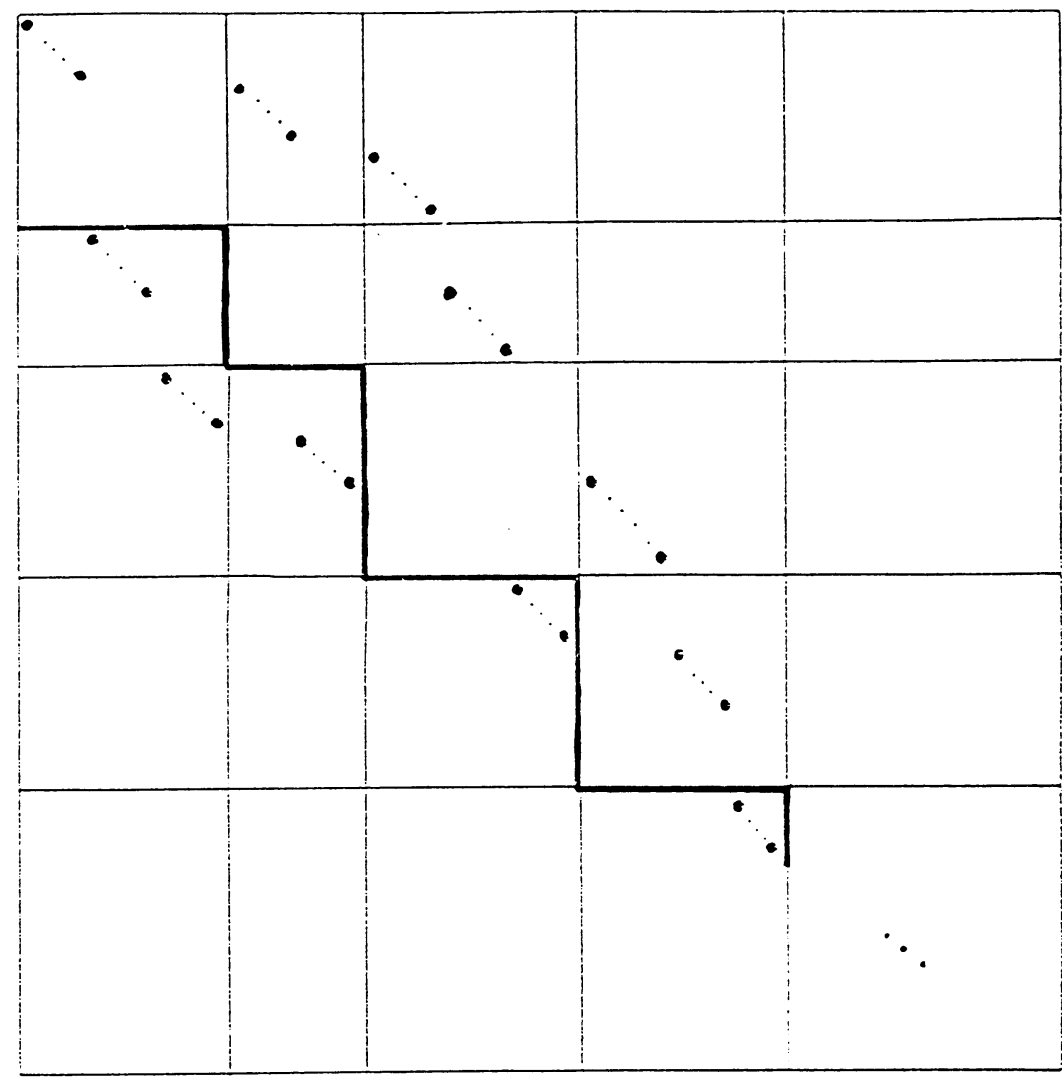

FIGURE 3. The graph of $\sigma_{d}$

(ii) We now construct the unique $\sigma_{d} \in S_{A}$. Since this $\sigma_{d}$ is a descending line on block-rows and block-columns, and also on $[i \preceq j]$ and $[i \succ j]$, the graph of $\sigma_{d}$ has the form shown in Figure 3.

It is now clear that this graph is uniquely determined by the set $A$. Namely, $r(l)$ (resp. $c(k))$ is the number of points $(i, \sigma(i))$ in $[i \succ j]$ that belong to the block-row $\eta^{*}(i)=l$ (resp. block-column $\eta^{*}(j)=k$ ), for $1 \leq k<l \leq r$.

Observe also that the conditions on $r(l)$ and $c(k)$ are necessary and sufficient conditions for being able to construct a graph as in Figure 3.

Definition 1.4. For $S_{A}=\left\{\sigma \in S^{(\eta)} \mid r_{\sigma}^{-}(l)=r(l), c_{\sigma}^{-}(k)=c(k)\right.$ for $1 \leq k<$ $l \leq r\}$ the unique $\sigma_{d}$ defined by Lemma 1(ii) is called a descending permutation, abbreviated as desc. perm.

The reason for calling $\sigma_{d}$ a descending permutation is clear from its graph, cf. Figure 3. We introduce some further notation:

Notation. For $\sigma_{d}$ a desc. perm. corresponding to $S_{A}$ as in Lemma 1, we denote

(i) $C_{\sigma_{d}}=S_{A}=\left\{\sigma \in S^{(\eta)} \mid r_{\sigma}^{-}(l)=r(l)\right.$ and $c_{\sigma}^{-}(k)=c(k)$ for $1 \leq k<$ $l \leq r\}$.

(ii) $\left\langle\sigma_{d}\right\rangle=\left\{\sigma \in C_{\sigma_{d}} \mid \sigma\right.$ is a descending line on $[i \preceq j]$ and on $\left.[i \succ j]\right\}$. 
(iii) For $\sigma_{0} \in\left\langle\sigma_{d}\right\rangle: C_{\sigma_{0}}^{0}=\left\{\sigma \in C_{\sigma_{d}} \mid \Gamma_{\sigma} \cap[i \preceq j]=\Gamma_{\sigma_{0}} \cap[i \preceq j]\right\}$.

(iv) For $\sigma_{1} \in C_{\sigma_{0}}^{0}, \sigma_{0} \in\left\langle\sigma_{d}\right\rangle: C_{\sigma_{1}}^{1}=\left\{\sigma \in C_{\sigma_{d}} \mid \Gamma_{\sigma} \cap[i \succ j]=\Gamma_{\sigma_{1}} \cap[i \succ j]\right\}$.

Lemma 2. The set $C_{\sigma_{d}}=\bigcup_{\sigma_{0} \in\left\langle\sigma_{d}\right\rangle} \bigcup_{\sigma_{1} \in C_{\sigma_{0}}^{0}} C_{\sigma_{1}}^{1}$ is a disjoint union.

Proof. Let $\sigma \in C_{\sigma_{d}}$, and denote by $i_{1}<\cdots<i_{m}$ the rows for which $(i, \sigma(i)) \in$ $[i \preceq j]$. We consider the permutation $\pi \in S_{m}$ such that

$$
\sigma\left(i_{\pi(1)}\right)<\sigma\left(i_{\pi(2)}\right)<\cdots<\sigma\left(i_{\pi(m)}\right)
$$

and define the unique $\sigma_{1}$ for which

$$
\begin{cases}\sigma_{1}\left(i_{k}\right)=\sigma\left(i_{\pi(k)}\right) & \text { for } 1 \leq k \leq m \\ \sigma_{1}(i)=\sigma(i) & \text { otherwise. }\end{cases}
$$

Then $\sigma_{1} \in C_{\sigma_{d}}$ such that $\sigma \in C_{\sigma_{1}}^{1}$, and $\sigma_{1}$ is a descending line on $[i \preceq j]$. Using similar arguments on $\sigma_{1}$ and $[i \succ j]$, we construct a unique $\sigma_{0} \in C_{\sigma_{d}}$ such that $\sigma_{1} \in C_{\sigma_{0}}^{0}$ and $\sigma_{0}$ is a descending line on $[i \succ j]$. But $\sigma_{0}$ is also a descending line on $[i \preceq j]$, since it coincides with $\sigma_{1}$; we therefore conclude that $\sigma_{0} \in\left\langle\sigma_{d}\right\rangle$.

\section{A COUNTING PROBLEM}

For the calculation of the genus zeta function of local hereditary orders, we need to consider the following counting problem; cf. the remark following Lemma 10.

For $\sigma \in S^{(\eta)}$ we consider two disjoint subsets $\sigma 1$ and $\sigma 2$ of the lattice $\{1, \ldots, n\}^{2}$ :

$$
\left\{\begin{array}{l}
\sigma 1=\left\{(i, j) \mid j>\sigma(i) \text { and } \sigma^{-1}(j)>i\right\} \\
\sigma 2=\left\{(i, j) \mid j>\sigma(i) \text { and } \sigma^{-1}(j)<i\right\} .
\end{array}\right.
$$

For $q \in \mathbf{N}$ we need to count how many matrices $\left(x_{i, j}\right) \in M_{n}(\mathbf{N})$ satisfy the properties

$$
(P)\left\{\begin{array}{l}
(P 1) x_{i, \sigma(i)}=1 \quad \text { (uniquely determined), } \\
(P 2) x_{i, j}=0 \quad \text { if } j<\sigma(i) \text { (uniquely determined), } \\
(P 3) x_{i, j}= \begin{cases}1, \ldots, q & \text { if }(i, j) \in[i \preceq j] \cap \sigma 1 \text { ( } q \text { possibilities), } \\
1, \ldots, q^{2} & \text { if }(i, j) \in[i \preceq j] \cap \sigma 2\left(q^{2}\right. \text { possibilities), }\end{cases} \\
(P 4) x_{i, j}= \begin{cases}1 & \text { if }(i, j) \in[i \succ j] \cap \sigma 1 \text { (uniquely determined), } \\
1, \ldots, q & \text { if }(i, j) \in[i \succ j] \cap \sigma 2 \text { (q possibilities). }\end{cases}
\end{array}\right.
$$

Remark. Denote $N_{\sigma}^{+}=\#([i \preceq j] \cap \sigma 2)$ and $N_{\sigma}^{-}=\#([i \succ j] \cap \sigma 1)$; then the number of matrices satisfying properties $(P)$ is $q^{N(\sigma)}$, where

$$
N(\sigma)=\frac{n(n-1)}{2}+N_{\sigma}^{+}-N_{\sigma}^{-} \in \mathbf{N} .
$$


Lemma 3. Let $\sigma_{d}$ be a desc. perm. with lower row and column indices $r_{\sigma}^{-}(l)$ and $c_{\sigma}^{-}(k), 1 \leq k<l \leq r$. For every $\sigma \in\left\langle\sigma_{d}\right\rangle$, there holds

$$
N_{\sigma}^{+}-N_{\sigma}^{-}=\sum_{2 \leq l \leq r} \frac{r_{\sigma}^{-}(l)\left(r_{\sigma}^{-}(l)+1\right)}{2} .
$$

Proof. For $1 \leq i_{0} \leq n$, denote

$$
\left\{\begin{array}{l}
J_{i_{0}}^{+}=\left\{j \mid \sigma^{-1}(j)<i_{0} \preceq j \text { and } j>\sigma\left(i_{0}\right)\right\}, \\
J_{i_{0}}^{-}=\left\{j \mid \sigma^{-1}(j)>i_{0} \succ j \text { and } j>\sigma\left(i_{0}\right)\right\} .
\end{array}\right.
$$

Then $N_{\sigma}^{+}-N_{\sigma}^{-}=\sum_{1 \leq i_{0} \leq n}\left(\# J_{i_{0}}^{+}-\# J_{i_{0}}^{-}\right)$.

We remark first that $i_{0} \preceq \sigma\left(i_{0}\right)$ implies $J_{i_{0}}^{+}=J_{i_{0}}^{-}=\varnothing$ :

(a) If $j \in J_{i_{0}}^{-}$, then $i_{0} \succ j>\sigma\left(i_{0}\right)$ implies $i_{0} \succ \sigma\left(i_{0}\right)$.

(b) If $j \in J_{i_{0}}^{+}$, then $j>\sigma\left(i_{0}\right) \succeq i_{0}>\sigma^{-1}(j)$ implies $j \succeq \sigma^{-1}(j)$, and since $\sigma$ is a desc. line on $[i \preceq j]$, we obtain that $i_{0}>\sigma^{-1}(j)$ implies $\sigma\left(i_{0}\right)>j$, contradicting $j \in J_{i_{0}}^{+}$.

We now fix $i_{0} \succ \sigma\left(i_{0}\right)$ and calculate $J_{i_{0}}^{-}, J_{i_{0}}^{+}$:

(a) Let $j \in J_{i_{0}}^{+}$; then $j \succeq i_{0} \succ \sigma\left(i_{0}\right)$, and the last condition can be omitted:

$$
J_{i_{0}}^{+}=\left\{j \mid \sigma^{-1}(j)<i_{0} \preceq j\right\} .
$$

Moreover, $i_{0} \approx \sigma^{-1}(j)$ and $j \in J_{i_{0}}^{+}$imply $i_{0} \succ \sigma\left(i_{0}\right)>j$, a contradiction; thus,

$$
J_{i_{0}}^{+}=\left\{j \mid \sigma^{-1}(j) \prec i_{0} \preceq j\right\} .
$$

Using Property (2) of $\S 1$, we conclude: if $i_{0} \succ \sigma\left(i_{0}\right)$, then

$$
\# J_{i_{0}}^{+}=\sum_{\eta^{*}\left(i_{0}\right) \leq k \leq r} \sum_{1 \leq l<\eta^{*}\left(i_{0}\right)} b_{\sigma}(l, k)=U_{\sigma}\left(\eta^{*}\left(i_{0}\right)\right) .
$$

(b) Let $j \in J_{i_{0}}^{-}$; then $j \prec i_{0}<\sigma^{-1}(j)$ implies $\sigma^{-1}(j) \succ j$. Since $\sigma$ is a descending line on $[i \succ j]$, we conclude that $i_{0}<\sigma^{-1}(j)$ is equivalent to $\sigma\left(i_{0}\right)<j$, and again the last condition can be omitted. We rewrite $J_{i_{0}}^{-}$as

$$
\begin{aligned}
J_{i_{0}}^{-} & =\left\{j \mid \sigma^{-1}(j)>i_{0} \succ j\right\} \\
& =\left\{j \mid \sigma^{-1}(j) \succeq i_{0} \succ j\right\} \backslash\left\{j \mid \sigma^{-1}(j) \approx i_{0} \succ j \text { and } \sigma^{-1}(j) \leq i_{0}\right\} .
\end{aligned}
$$

We now calculate the cardinality of the last set,

$$
\mathrm{Sub}=\left\{j \mid \sigma^{-1}(j) \approx i_{0} \succ j \text { and } \sigma^{-1}(j) \leq i_{0}\right\} .
$$

Since $i_{0} \geq \sigma^{-1}(j) \approx i_{0}$ implies $\sigma\left(i_{0}\right) \geq j$, which in turn implies $i_{0} \succ j$, we can omit the second condition; thus, Sub $=\left\{j=\sigma(i) \mid i_{0} \approx i\right.$ and $\left.i_{0} \geq i\right\}$. Therefore, \# Sub $=i_{0}-S_{\eta}\left(\eta^{*}\left(i_{0}\right)\right)$, and as in (a) we find

$$
\# J_{i_{0}}^{-}=U_{\sigma}\left(\eta^{*}\left(i_{0}\right)\right)-\left[i_{0}-S_{n}\left(\eta^{*}\left(i_{0}\right)\right)\right]=\# J_{i_{0}}^{+}-\left[i_{0}-S_{n}\left(\eta^{*}\left(i_{0}\right)\right)\right] .
$$


So, finally,

$$
\begin{aligned}
N_{\sigma}^{+}-N_{\sigma}^{-} & =\sum_{1 \leq i_{0} \leq n} J_{i_{0}}^{+}-J_{i_{0}}^{-}=\sum_{\substack{1 \leq i_{0} \leq n \\
i_{0} \succ \sigma\left(i_{0}\right)}}\left[i_{0}-S_{\eta}\left(\eta^{*}\left(i_{0}\right)\right)\right] \\
& =\sum_{2 \leq l \leq r} \sum_{1 \leq s \leq r_{\sigma}^{-}(l)} s=\sum_{2 \leq l \leq r} \frac{r_{\sigma}^{-}(l)\left(r_{\sigma}^{-}(l)+1\right)}{2} .
\end{aligned}
$$

This completes the proof of Lemma 3.

Definition 2.1. For $0 \leq l \leq m$, define the Gaussian polynomials

$$
\left[\begin{array}{c}
0 \\
m
\end{array}\right]=1, \quad\left[\begin{array}{c}
l \\
m
\end{array}\right]=\frac{\left(q^{m}-1\right) \cdots\left(q^{m-l+1}-1\right)}{\left(q^{l}-1\right) \cdots(q-1)}, \quad[m]=\left[\begin{array}{c}
1 \\
m
\end{array}\right] .
$$

Lemma 4. We have

$$
\left[\begin{array}{c}
l \\
m
\end{array}\right]=\sum_{0 \leq j \leq m-l} q^{l j} \cdot\left[\begin{array}{c}
l-1 \\
m-j-1
\end{array}\right] \text { if } l>0 .
$$

Proof. For $l=1$, the identity is evident, and for $l>1$ it can easily be proved by induction on $l$.

Lemma 5. For $I=\left\{i_{1}<\cdots<i_{l}<\cdots<i_{m}\right\}$ and $\sigma_{0} \in S_{n}$ with $\sigma_{0}\left(i_{1}\right)<\cdots<$ $\sigma_{0}\left(i_{m}\right)$, consider

$$
C_{\sigma_{0}}^{(l, m)}=\left\{\sigma \in S_{n} \mid \sigma(i)=\sigma_{0}(i) \text { if } i \notin I \text { and }\left\{\begin{array}{c}
\sigma\left(i_{1}\right)<\cdots<\sigma\left(i_{l}\right) \\
\sigma\left(i_{l+1}\right)<\cdots<\sigma\left(i_{m}\right)
\end{array}\right\}\right\} .
$$

Then

$$
\sum_{\sigma \in C_{\sigma_{0}}^{(l, m)}} q^{N(\sigma)}=q^{N\left(\sigma_{0}\right)} \cdot\left[\begin{array}{c}
l \\
m
\end{array}\right]
$$

Proof. We prove this by induction on $l$.

(a) $l=0: C_{\sigma_{0}}^{(0, m)}=\left\{\sigma_{0}\right\}$, so the statement follows.

(b) Assume that the statement holds for $l^{\prime}<l$. As illustrated in Figures 4(a) and $4(\mathrm{~b})$, for $0 \leq j \leq m-l$ we consider $\sigma^{(j)}$, the unique $\sigma \in C_{\sigma_{0}}^{(l, m)}$ for which

$$
\underbrace{\sigma\left(i_{l+1}\right)<\cdots<\sigma\left(i_{l+j+1}\right)}_{j}<\sigma\left(i_{1}\right)<\underbrace{\overbrace{\sigma\left(i_{2}\right)<\cdots<\sigma\left(i_{l}\right)}^{l-1}<\sigma\left(i_{l+j+2}\right)<\cdots<\sigma\left(i_{m}\right)}_{m-j-1} .
$$

It is clear that $C_{\sigma_{0}}^{(l, m)}=\bigcup_{0 \leq j \leq m-l} C_{\sigma^{(j)}}^{(l-1, m-j-1)}$ (a disjoint union); indeed,

$$
C_{\sigma^{(j)}}^{(l-1, m-j-1)}=\left\{\sigma \in C_{\sigma_{0}}^{(l, m)} \mid \sigma\left(i_{1}\right)=\sigma^{(j)}\left(i_{1}\right)\right\} .
$$

Moreover, it follows from Figures $4\left(\right.$ a) and $4\left(\right.$ b) that $N\left(\sigma^{(j)}\right)=N\left(\sigma_{0}\right)+l j$. From the induction hypothesis we obtain

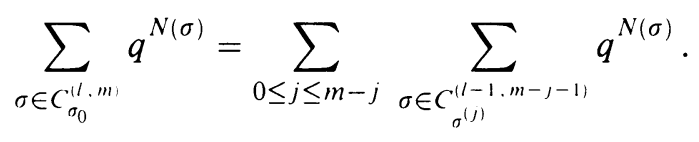




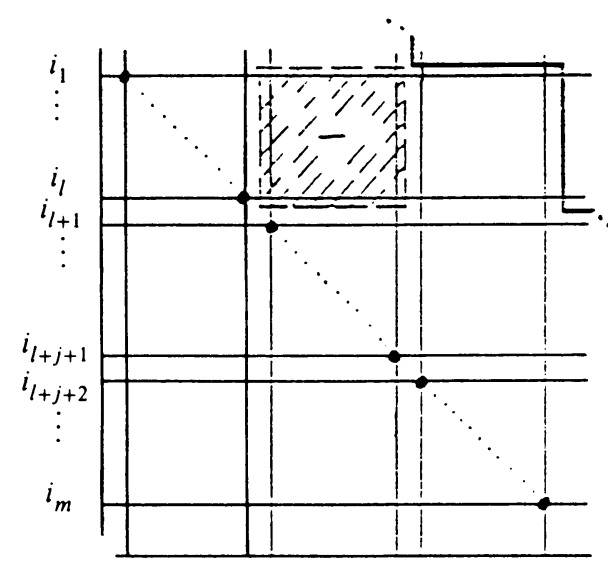

FIGURE $4(\mathrm{a}) . \sigma_{0}$

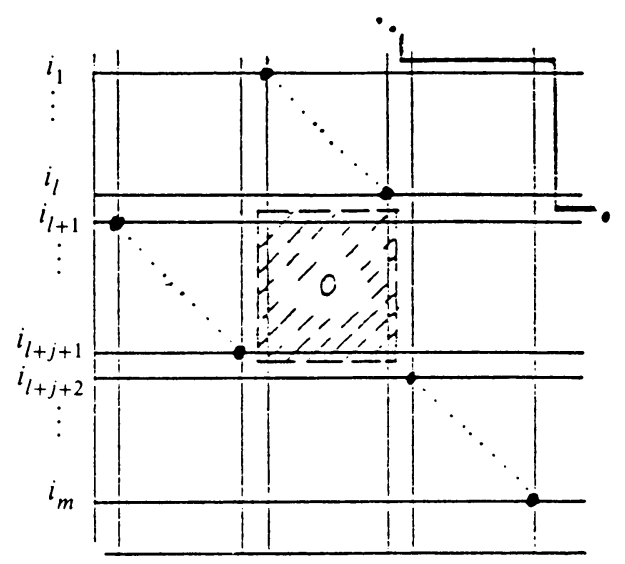

FIGURE 4(b). $\sigma^{(j)}$

We thus conclude

$$
\sum_{\sigma \in C_{\sigma_{0}}^{(l, m)}} q^{N(\sigma)}=q^{N\left(\sigma_{0}\right)} \sum_{0 \leq j \leq m-l} q^{l j} \cdot\left[\begin{array}{c}
l-1 \\
m-j-1
\end{array}\right]=q^{N\left(\sigma_{0}\right)} \cdot\left[\begin{array}{c}
l \\
m
\end{array}\right] .
$$

Theorem 6. For a desc. perm. $\sigma_{d}$ corresponding to $r_{\sigma}^{-}(l)$ and $c_{\sigma}^{-}(k), 1 \leq k<$ $l \leq r$, we have

$$
\begin{aligned}
& \text { (i) } \sum_{\sigma \in C_{\sigma_{0}}^{0}} q^{N(\sigma)}=q^{N\left(\sigma_{0}\right)} \prod_{2 \leq l \leq r}\left[\begin{array}{c}
r_{\sigma}^{-}(l) \\
U_{\sigma}(l)
\end{array}\right] \text { for every } \sigma_{0} \in\left\langle\sigma_{d}\right\rangle ; \\
& \text { (ii) } \sum_{\sigma \in C_{\sigma_{1}}^{\prime}} q^{N(\sigma)}=q^{N\left(\sigma_{1}\right)} \prod_{2 \leq l \leq r}\left[\begin{array}{c}
r_{\sigma}^{+}(l) \\
U_{\sigma}(l)+r_{\sigma}^{+}(l)
\end{array}\right] \text { for every } \sigma_{1} \in C_{\sigma_{0}}^{0} .
\end{aligned}
$$

Proof. (i) Recall that $C_{\sigma_{0}}^{0}=\left\{\sigma \in C_{\sigma_{d}} \mid \Gamma_{\sigma_{0}} \cap[i \preceq j]=\Gamma_{\sigma} \cap[i \preceq j]\right\}$. Denote $I_{2}=\left\{i \mid \eta^{*}(i) \geq 2>\eta^{*}(\sigma(i))\right\}=\left\{i_{1}<\cdots<i_{U_{\sigma}(2)}\right\}$ and observe that $\left\{i_{1}<\right.$ $\left.\cdots<i_{r_{\sigma}^{-}(2)}\right\}=\left\{i \in I_{2} \mid \eta^{*}(i)=2\right\}$. Since $\sigma_{0}\left(i_{1}\right)<\cdots<\sigma_{0}\left(i_{U_{\sigma}(2)}\right)$, we can apply Lemma 5 to obtain

$$
\sum_{\sigma \in C_{\sigma_{0}}^{(2)}} q^{N(\sigma)}=q^{N\left(\sigma_{0}\right)} \cdot\left[\begin{array}{l}
r_{\sigma}^{-}(2) \\
U_{\sigma}(2)
\end{array}\right]
$$

where $C_{\sigma}^{(k)}=C_{\sigma}^{\left(r_{\sigma}^{-}(k), l_{\sigma}^{\prime}(k)\right)}$ for $2 \leq k \leq r$. To continue this procedure, we renumber the rows and consider $I_{3}=\left\{i \mid \eta^{*}(i) \geq 3>\eta^{*}(\sigma(i))\right\}=\left\{i_{1}<\cdots<\right.$ $\left.i_{U_{\sigma}^{\prime}(3)}\right\}$ with $\left\{i_{1}<\cdots<i_{r_{\sigma}^{-}(3)}\right\}=\left\{i \in I_{3} \mid \eta^{*}(i)=3\right\}$. For every $\sigma_{1} \in C_{\sigma_{0}}^{(2)}$ we find $\sigma_{1}\left(i_{1}\right)<\cdots<\sigma_{1}\left(i_{L_{\sigma}^{\prime}(3)}\right)$, so we can apply Lemma 5 and find

$$
\sum_{\sigma \in C_{\sigma_{1}}^{(3)}} q^{N(\sigma)}=q^{N\left(\sigma_{1}\right)} \cdot\left[\begin{array}{l}
r_{\sigma}^{-}(3) \\
U_{\sigma}(3)
\end{array}\right]
$$


Similar calculations can be made for $\sigma_{2} \in C_{\sigma_{1}}^{(3)}, \ldots, \sigma_{r-1} \in C_{\sigma_{r-2}}^{(r)}$, and we note that

$$
C_{\sigma_{0}}^{0}=\bigcup_{\sigma_{1} \in C_{\sigma_{0}}^{(2)}} \bigcup_{\sigma_{2} \in C_{\sigma_{1}}^{(3)}} \cdots \bigcup_{\sigma_{r-1} \in C_{\sigma_{r-2}}^{(r)}}\left\{\sigma_{r-1}\right\}
$$

is a disjoint union. We finally obtain

$$
\sum_{\sigma \in C_{\sigma_{0}}^{0}} q^{N(\sigma)}=q^{N\left(\sigma_{0}\right)} \prod_{2 \leq k \leq r}\left[\begin{array}{c}
r_{\sigma}^{-}(k) \\
U_{\sigma}(k)
\end{array}\right] .
$$

(ii) Recall that $C_{\sigma_{1}}^{1}=\left\{\sigma \in C_{\sigma_{d}} \mid \Gamma_{\sigma_{1}} \cap[i>j]=\Gamma_{\sigma} \cap[i>j]\right\}$. Denote $J_{2}=\left\{i \mid \eta^{*}(i) \leq 2 \leq \eta^{*}(\sigma(i))\right\}=\left\{i_{1}<\cdots<i_{U_{\sigma}^{\prime}(2)+r_{\sigma}^{+}(2)}\right\}$. Since $\sigma_{1}\left(i_{1}\right)<\cdots<$ $\sigma_{1}\left(i_{L_{\sigma}^{\prime}(2)+r_{\sigma}^{+}(2)}\right)$, we can apply Lemma 5 to obtain

$$
\sum_{\sigma \in D_{\sigma_{1}}^{(2)}} q^{N(\sigma)}=q^{N\left(\sigma_{1}\right)} \cdot\left[\begin{array}{c}
r_{\sigma}^{+}(2) \\
U_{\sigma}(2)+r_{\sigma}^{+}(2)
\end{array}\right]
$$

where $D_{\sigma}^{(k)}=C_{\sigma}^{\left(r_{\sigma}^{+}(k), l_{\sigma}^{(}(k)+r_{\sigma}^{+}(k)\right)}$ for $1 \leq k \leq r$. We continue the proof as in (i), and the assertion easily follows.

\section{THE GENUS ZETA FUNCTION OF A LOCAL HEREDITARY ORDER}

Let $R$ be a discrete valuation ring in a local field $K$ with prime ideal $P$. We will consider hereditary $R$-orders in a central simple $K$-algebra $A=M_{n}(D)$. The valuation $v$ has a unique extension $v_{D}$ in the skew field $D$. We denote by $\Delta$ the unique maximal $R$-order in $D$ and by $n=\Delta \bar{\pi}$ the unique two-sided prime ideal in $\Delta$. Then $\#(\Delta / \mu)=q$ with $q$ finite; we refer to [11] for a more detailed description.

A maximal $R$-order in $A$ is isomorphic to $M_{n}(\Delta)$. For the hereditary $R$ orders in $A$ we have

Theorem 7. A hereditary $R$-order in $A$ is defined up to isomorphism by its local type $r$ and local invariants $\eta=\left(n_{1}, \ldots, n_{r}\right), n_{i} \neq 0$, with $S_{\eta}(r)=n$, namely

$$
\Theta=\Theta^{\eta}=\left\{x \in M_{n}(\Delta) \mid x_{i, j} \in \rho \text { if } \eta^{*}(i)>\eta^{*}(j)\right\},
$$

where $\eta^{*}$ is the block map associated with the r-tuple $\eta$.

Proof. Cf. [11, p. 358].

Remark. The local invariants $\eta$ are determined up to a cyclic permutation.

Definition 3.1. The genus zeta function of a local $R$-order $\Theta$ is

$$
Z_{\Theta}(s)=\sum(\Theta: \mathscr{L})^{-s}, \quad \mathfrak{R} e(s)>1,
$$

where the sum ranges over the free ideals $\mathscr{L}=\Theta x, x \in A^{*}$ (the units of $A$ ), which are integral (i.e., $\mathscr{L} \subset \Theta)$ and $(\Theta: \mathscr{L})=\#(\Theta / \mathscr{L})$.

Remark. One has $(\Theta: \Theta x)=|x|^{-1}$, where $|x|$ is the module of $x$, cf. [1]. 
Theorem 8. For a maximal $R$-order $\Lambda$ in $A$ we have

$$
Z_{\Lambda}(s)=\zeta_{\Lambda}(s)=\prod_{0 \leq j \leq n-1}\left(1-q^{j-n s}\right)^{-1} .
$$

Proof. We sketch the main steps of the proof; details can be found in [1, Chapter 3.3].

Note first that $h(\Lambda)=1$, where $h(\Lambda)$ is the class number of a local maximal order $\Lambda$, so the zeta function of $\Lambda$ coincides with its genus zeta function. Furthermore, $\zeta_{\Lambda}(s)=\sum^{\prime}|x|^{s}$, where $x$ ranges over a full set of representatives of $\Lambda^{*} \backslash \Lambda \cap A^{*}$. We can assume that $\Lambda=M_{n}(\Delta)$; then every class in $\Lambda^{*} \backslash \Lambda \cap A^{*}$ has a unique representative in "Hermite-normal form", i.e., $x=\left(a_{i, j}\right)$ with

$$
\begin{cases}a_{i, i}=\bar{\pi}^{\lambda_{i}}, & \lambda_{i} \in \mathbf{N}, \\ a_{i, j}=0 & \text { if } j<i, \\ a_{i, j} \in \Delta / \bar{\pi}^{\lambda_{j}} \Delta & \text { if } j>i .\end{cases}
$$

Observe that these "Hermite-normal forms" are upper triangular. The number of "Hermite-normal forms" with fixed values of $\lambda=\left(\lambda_{1}, \ldots, \lambda_{n}\right)$ is

$$
q^{0 \cdot \lambda_{1}+1 \cdot \lambda_{2}+\cdots+(n-1) \cdot \lambda_{n}}=\prod_{1 \leq j \leq n} q^{(j-1) \lambda}
$$

For these $x$, we have $|x|=q^{-\left(\lambda_{1}+\cdots+\lambda_{n}\right) \cdot n}$. We conclude that

$$
\zeta_{\Lambda}(s)=\sum_{i \in \mathbf{N}^{n}}\left(\prod_{1 \leq j \leq n} q^{j-1-n s}\right)^{i,}=\prod_{0 \leq j<n}\left(1-q^{j-n s}\right)^{-1}
$$

This completes the proof.

We recall a result of C. J. Bushnell and I. Reiner on the genus zeta function of local $R$-orders in central simple algebras:

Theorem 9. If $\Theta \subset \Lambda$ are $R$-orders in $A$ with $\Lambda$ maximal, then

$$
Z_{\Theta}(s)=\zeta_{\Lambda}(s) \cdot f_{\Theta}\left(q^{-s}\right) \text { with } f_{\Theta}\left(q^{-s}\right) \in \mathbf{Z}\left[q^{-s}\right]
$$

Proof. Cf. [1, Theorem 1].

Our aim is to determine the polynomial $f_{\Theta}$ for hereditary $R$-orders in $A$.

Lemma 10. Let $\Theta=\Theta^{\prime \prime}$ be the standard hereditary order with local invariants $\eta$, cf. Theorem 7; then every class in $\Theta^{*} \backslash \Theta \cap A^{*}$ can be uniquely represented by $a$ " $\Theta$-normal form" $x$. Here, $x=\left(a_{i, j}\right) \in \Theta$ is a " $\Theta$-normal form" belonging to $\sigma \in S^{(\eta)}$ and $\lambda \in \mathbf{N}^{n}$ if and only if

$$
\left\{\begin{array}{l}
(n 1) a_{i, \sigma(i)}=\bar{\pi}^{\lambda_{\sigma(1)},} \\
(n 2) a_{i, j}=0 \quad \text { if } j<\sigma(i), \\
(n 3) a_{i, j} \in\left\{\begin{array}{l}
\Delta / \mu^{i,} \quad \text { if }(i, j) \in \sigma 1, \\
\Delta / p^{i,+1} \quad \text { if }(i, j) \in \sigma 2,
\end{array}\right. \\
(n 4) \bar{\pi} \mid a_{i, j} \quad \text { if }(i, j) \in[i>j] .
\end{array}\right.
$$


Proof. Cf. [9, p. 35] for a detailed proof. Using the same techniques as for the reduction to "Hermite-normal forms", we get these " $\Theta$-normal forms" if we consider that the elementary transformations corresponding to left multiplication with matrices $\varepsilon$ are 'allowed' if and only if $\varepsilon \in \Theta^{*}$. For $\varepsilon=P_{i j}$, the permutation of the $i$ th and the $j$ th row, we have $\varepsilon \in \Theta^{*}$ if and only if $\eta^{*}(i)=\eta^{*}(j)$. For $\varepsilon=T_{i j}(y)$, replacing the $i$ th row by the $i$ th row $+y$ times the $j$ th row, we have $\varepsilon \in \Theta^{*}$ precisely if $i \prec j$ or $\bar{\pi} \mid y$.

Remark. If $\lambda_{1}=\cdots=\lambda_{n}=1$, then the " $\Theta^{\eta}$-normal forms" corresponding to $\sigma \in S^{(\eta)}$ are bijectively in correspondence with the matrices in $(P)$ of $\S 2$. If $\sigma=1_{n}$, the identity, then the " $\Theta^{\eta}$-normal forms" corresponding to $\sigma$ are the "Hermite-normal forms". Moreover, if $x \in \Theta^{\eta}$ is a " $\Theta^{\eta}$-normal form" corresponding to $\sigma \in S^{(\eta)}$ and $\lambda \in \mathbf{N}^{n}$, then clearly $\lambda_{\sigma(i)}>0$ if $i \succ \sigma(i)$. We conclude that the number of " $\Theta^{\eta}$-normal forms" corresponding to $\sigma \in S^{(\eta)}$ and $\lambda \in \mathbf{N}^{n}$, with $\lambda_{\sigma(i)}>0$ if $i \succ \sigma(i)$, is

$$
q^{N_{\sigma}^{+}-N_{\sigma}^{-}} \cdot \prod_{1 \leq j \leq n} q^{(j-1) \cdot \lambda_{j}} .
$$

Theorem 11. The genus zeta function of a hereditary $R$-order $\Theta \cong \Theta^{\eta}$ is

$$
Z_{\boldsymbol{\Theta}}(s)=\zeta_{\Lambda}(s) \cdot f_{\boldsymbol{\Theta}}\left(q^{-n s}\right),
$$

where $f_{\Theta}(Y)=\sum_{\sigma \text { a desc. perm. }} C(\sigma) \cdot q^{\mathrm{pow}(\sigma)} \cdot Y^{k(\sigma)}$ with $Y=q^{-n s}$ and

$$
\left\{\begin{array}{l}
C(\sigma)=\prod_{2 \leq l \leq r}\left[\begin{array}{l}
r_{\sigma}^{-}(l) \\
U_{\sigma}(l)
\end{array}\right] \cdot\left[\begin{array}{c}
r_{\sigma}^{+}(l) \\
U_{\sigma}(l)+r_{\sigma}^{+}(l)
\end{array}\right] \cdot \prod_{1 \leq k<r}\left[\begin{array}{c}
c_{\sigma}^{-}(k) \\
n_{k}
\end{array}\right], \\
\operatorname{pow}(\sigma)=\frac{1}{2}\left\{\sum_{2 \leq l \leq r} r_{\sigma}^{-}(l)^{2}+\sum_{1 \leq k<r} c_{\sigma}^{-}(k)^{2}\right\}+\sum_{1 \leq k<r} S_{\eta}(k-1) \cdot c_{\sigma}^{-}(k), \\
k(\sigma)=\# I_{\sigma} \text { with } I_{\sigma}=\left\{j \mid \sigma^{-1}(j) \succ j\right\} .
\end{array}\right.
$$

Proof. As in Theorem 8, we can assume that $\Theta=\Theta^{\eta}$, and we find $Z_{\boldsymbol{\Theta}}(s)=$ $\sum^{\prime}|x|^{s}$, where $x$ runs over the " $\Theta^{\eta}$-normal forms", so

$$
Z_{\boldsymbol{\Theta}}(s)=\sum_{\sigma \in S^{(n)}} q^{N_{\sigma}^{+}-N_{\sigma}^{-}} \sum_{\substack{\lambda \in \mathbf{N}^{n} \\ \lambda_{j}>0 \text { if } j \in I_{\sigma}}} \prod_{1 \leq j \leq n}\left(q^{j-1-n s}\right)^{\lambda_{j}} .
$$

Fix $\sigma_{0} \in\left\langle\sigma_{d}\right\rangle$ with $\sigma_{d}$ a descending permutation. Note that for $\sigma \in \bigcup_{\sigma_{1} \in C_{\sigma_{0}}^{0}} C_{\sigma_{1}}^{1}$ we have $I_{\sigma}=I_{\sigma_{0}}$. Using Theorem 6 , we calculate

$$
\begin{aligned}
B\left(\sigma_{0}\right) & =\sum_{\sigma_{1} \in C_{\sigma_{0}}^{0}} \sum_{\sigma \in C_{\sigma_{1}}^{1}} q^{N_{\sigma}^{+}-N_{\sigma}^{-}} \\
& =q^{N_{\sigma_{0}}^{+}-N_{\sigma_{0}}^{-}} \cdot \prod_{2 \leq l \leq r}\left[\begin{array}{c}
r_{\sigma}^{-}(l) \\
U_{\sigma}(l)
\end{array}\right] \cdot\left[\begin{array}{c}
r_{\sigma}^{+}(l) \\
U_{\sigma}(l)+r_{\sigma}^{+}(l)
\end{array}\right] .
\end{aligned}
$$


From Lemma 3 we recall $N_{\sigma_{0}}^{+}-N_{\sigma_{0}}^{-}=\frac{1}{2} \sum_{2 \leq l \leq r} r_{\sigma}^{-}(l)\left(r_{\sigma}^{-}(l)+1\right)$ for every $\sigma_{0} \in\left\langle\sigma_{d}\right\rangle ;$ thus $B\left(\sigma_{0}\right)=B\left(\sigma_{d}\right)$. Using Lemma 2 , we find

$$
Z_{\boldsymbol{\Theta}}(s)=\sum_{\sigma_{d} \text { a desc. perm. }} B\left(\sigma_{d}\right) \sum_{\sigma_{0} \in\left\langle\sigma_{d}\right\rangle} \sum_{\substack{\lambda \in \mathbb{N}^{n} \\ \lambda_{j}>0 \text { if } j \in I_{\sigma}}} \prod_{1 \leq j \leq n}\left(q^{j-1-n s}\right)^{\lambda_{j}} .
$$

Observe that for $I \subset\{1, \ldots, n\}$ we have

$$
\sum_{\substack{\lambda \in \mathbf{N}^{n} \\ \lambda_{j}>0 \text { if } j \in I}} f(s)=\sum_{\lambda \in \mathbf{N}^{n}} f(s)-\sum_{j \in I} \sum_{\substack{\lambda \in \mathbf{N}^{n} \\ \lambda_{j}=0}} f(s)+\cdots+(-1)^{\# I} \sum_{\substack{\lambda \in \mathbf{N}^{n} \\ \lambda_{j}=0 \text { if } j \in I}} f(s) .
$$

We apply this to $f(s)=\prod_{1 \leq j \leq n}\left(q^{j-1-n s}\right)^{\lambda_{j}}$ and $I=I_{\sigma}$. This yields

$$
\sum_{\substack{\lambda \in \mathbb{N}^{n} \\ \lambda_{j}>0 \text { if } j \in I_{\sigma}}} f(s)=\prod_{1 \leq j \leq n}\left(1-q^{j-1-n s}\right)^{-1} \cdot \prod_{j \in I_{\sigma}} q^{j-1-n s} .
$$

Substituting this in the expression for $Z_{\boldsymbol{\theta}}(s)$, we find

$$
\zeta_{\Theta}(s)=\zeta_{\Lambda}(s) \cdot \sum_{\sigma_{d} \text { a desc. perm. }} B\left(\sigma_{d}\right)\left(\sum_{\sigma \in\left\langle\sigma_{d}\right\rangle} \prod_{j \in I_{\sigma}} q^{j-1}\right) \cdot Y^{k(\sigma)} .
$$

Now we calculate

$$
\sum_{\sigma \in\left\langle\sigma_{d}\right\rangle} \prod_{j \in I_{\sigma}} q^{j-1}
$$

Let $\sigma_{m} \in\left\langle\sigma_{d}\right\rangle$ be uniquely determined by

$$
I_{\sigma_{m}}=\left\{S_{\eta}(k-1)+j \mid 1 \leq k<r \text { and } 1 \leq j \leq c_{\sigma}^{-}(k)\right\} .
$$

Then the exact power of $q$ dividing (1) corresponds to the contribution of $\sigma_{m}$ and is given by

$$
q^{N}=\prod_{j \in I_{\sigma_{m}}} q^{j-1} \quad \text { with } N=\sum_{1 \leq k<r} c_{\sigma}^{-}(k) \cdot S_{\eta}(k-1)+\frac{c_{\sigma}^{-}(k)\left(c_{\sigma}^{-}(k)-1\right)}{2} .
$$

To calculate (1), we use the same techniques as in the proof of Theorem 6: Denote $J_{k}=\left\{i_{1}<\cdots<i_{n_{k}}\right\}=\left\{i \mid \eta^{*}\left(\sigma_{m}(i)\right)=k\right\}$ and note that $\left\{i_{1}<\cdots<\right.$ $\left.i_{c_{\sigma}^{+}(k)}\right\}=\left\{i \mid \eta^{*}\left(\sigma_{d}(i)\right)=k \succeq i\right\}$. Let $C_{\sigma_{m}}^{(k)}=C_{\sigma_{m}}^{\left(c_{\sigma}^{+}(k), n_{k}\right)}$ be defined as in Lemma 5:

$$
C_{\sigma_{m}}^{(k)}=\left\{\sigma \in\left\langle\sigma_{d}\right\rangle \mid \sigma(i)=\sigma_{m}(i) \text { if } i \notin J_{k} \text { and }\left\{\begin{array}{c}
\sigma\left(i_{1}\right)<\cdots<\sigma\left(i_{c_{\sigma}^{+}(k)}\right) \\
\sigma\left(i_{c_{\sigma}^{+}(k)+1}\right)<\cdots<\sigma\left(i_{n_{k}}\right)
\end{array}\right\}\right. \text {. }
$$

Since the powers of $q$ in (1) are exactly the same as the powers of $q$ in Lemma 5 , we can extend the result:

$$
\sum_{\sigma \in C_{\sigma_{m}}^{(k)}} \prod_{j \in I_{\sigma}} q^{j-1}=q^{N} \cdot\left[\begin{array}{c}
c_{\sigma}^{+}(k) \\
n_{k}
\end{array}\right]
$$


Writing $\left\langle\sigma_{d}\right\rangle$ as a disjoint union over $C_{\sigma}^{(k)}$, we conclude that

$$
\text { (1) }=q^{N} \cdot \prod_{1 \leq k \leq r}\left[\begin{array}{c}
c_{\sigma}^{+}(k) \\
n_{k}
\end{array}\right] \text {, }
$$

and the theorem follows.

Remarks. (1) The result is technically complicated but it can be used to calculate the genus zeta function explicitly. Namely, generate all sets $\left\{b_{\sigma}(l, k) \mid 1 \leq k<\right.$ $l \leq r\}$ for which the following conditions are satisfied:

$$
\left\{\begin{array}{l}
\text { (i) } \sum_{1 \leq j<l} b_{\sigma}(l, j) \leq n_{l} \text { and } \sum_{k<i \leq r} b_{\sigma}(i, k) \leq n_{k} \text { for } 1 \leq k<l \leq r \\
\text { (ii) } b_{\sigma}(l, k) \neq 0 \Rightarrow b_{\sigma}(l+1, j)=0 \text { for all } j<k .
\end{array}\right.
$$

These conditions imply that there exists a unique descending permutation $\sigma$ with these block numbers. Then we calculate $k(\sigma), C(\sigma)$, and $\operatorname{pow}(\sigma)$ using Theorem 11. This computation can be done by a computer.

(2) We have a check, to detect errors in the program, since

$$
f_{\Theta}(1)=\frac{\prod_{1 \leq i \leq n}[i]}{\prod_{1 \leq i \leq r} \prod_{1 \leq j \leq n_{i}}[j]} .
$$

This follows from the idele description for $Z_{\boldsymbol{\Theta}}(s)$ and the functional equation, see $[1,3,9]$. We find that

$$
f_{\Theta}(1)=\frac{Z_{\Theta}(0)}{\zeta_{\Lambda}(0)}=\frac{\mu^{*}\left(\Theta^{\eta^{*}}\right)}{\mu^{*}\left(\Lambda^{*}\right)}
$$

which can be calculated for hereditary orders, see [13, 9].

In the appendix we give some examples calculated with the Siemens mainframe computer (BS2000 Operating System).

\section{THE GENUS ZETA FUNCTION OF MINIMAL HEREDITARY ORDERS AND $q$-EULERIAN POLYNOMIALS}

L. Carlitz introduced the notion of $q$-Eulerian polynomials $A_{n, k}(q)$ for $0<$ $k \leq n$, with the property that, evaluated at $q=1$, one obtains the Eulerian numbers, cf. [6]. These polynomials are defined by the recursion formula

$$
A_{n, k}(q)=[n+1-k] \cdot A_{n-1, k-1}(q)+q^{n-k}[k] \cdot A_{n-1, k}(q) \text {. }
$$

Denote by $B_{n, k}(q), 0 \leq k<n$, the coefficient of $Y^{k}$ in $f_{\Theta}(Y)$, with $\Theta=\Theta^{\eta}$ the minimal hereditary order, i.e., $\eta=(1, \ldots, 1)$. The referee informed us of a quite unexpected relationship between the $B_{n, k}(q)$ and the $A_{n, k}(q)$.

After we calculated examples up to $n=20$, we conjecture that the exact relation is

$$
A_{n, n-k}(q)=B_{n, k}(q) \text {. }
$$

We are not able to match the definition of $B_{n, k}(q)$ with the known interpretations for $A_{n, k}(q)$, cf. [6]. So we think the only way to prove the relation is to show that $B_{n, k}(q)$ satisfies the recursion formula. From Theorem 11 we now 
deduce the expression $(C)$ below for $B_{n, k}(q)$ which only involves combinatorial calculations.

For $\eta=(1, \ldots, 1)$ we find $S^{(\eta)}=S_{n}$ (namely $\eta^{*}(i)=i$ for every $\left.i\right)$, so Theorem 11 yields

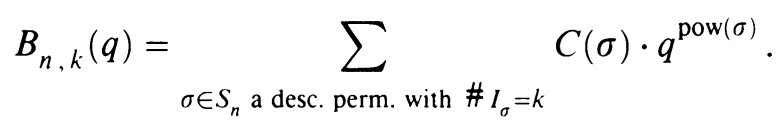

For $\sigma \in S_{n}$, let $I_{\sigma}=\left\{j_{1}<\cdots<j_{k}\right\}$ with $1 \leq j_{m}=\sigma\left(i_{m}\right)<i_{m} \leq n$ and $k=\# I_{\sigma}$. Restricting to a desc. perm. $\sigma$ yields $i_{1}<\cdots<i_{k}$ and such a $\sigma$ is completely determined by the $k$ couples $\left(i_{m}, j_{m}\right)$.

We calculate now $C(\sigma)$ and $\operatorname{pow}(\sigma)$ in terms of $\left(i_{m}, j_{m}\right): \operatorname{pow}(\sigma)=j_{1}+$ $\cdots+j_{k}$. Moreover, $U_{\sigma}(l)=\#\{i \mid \sigma(i)<l \leq i\}=\#\left\{m \mid j_{m}<l \leq i_{m}\right\}$. We introduce the notation $V_{\left(i_{m}, j_{m}\right)}(l)=\#\left\{m \mid j_{m}<l<i_{m}\right\}+1$. Since $r_{\sigma}^{-}\left(i_{m}\right)=1$ and $r_{\sigma}^{+}\left(i_{m}\right)=0$ for $1 \leq m \leq k$, and $r_{\sigma}^{-}(l)=0$ and $r_{\sigma}^{+}(l)=1$ otherwise, we find $V_{\left(i_{m}, j_{m}\right)}(l)=U_{\sigma}(l)+r_{\sigma}^{+}(l)$.

With $n_{j}=1$ we obtain $C(\sigma)=\prod_{1 \leq l \leq n}\left[V_{\left(i_{m}, j_{m}\right)}(l)\right]$ and thus

$$
B_{n, k}(q)=\sum_{\left(i_{m}, j_{m}\right)} q^{j_{1}+\cdots+j_{k}} \prod_{1 \leq l \leq n}\left[V_{\left(i_{m}, j_{m}\right)}(l)\right] .
$$

We remark that the recursion formula has no natural interpretation. Using the expression $(C)$, we could prove the recursion for $k \leq 2$, but we were not able to prove it in the general case. The conjecture $(C)$ is proven by D. Foata and D. Zeilberger, in Denert's permutation statistic is indeed Euler-Mahonian, preprint. Moreover, the "problem" only occurs for minimal hereditary orders. So, maybe a generalization of $q$-Eulerian polynomials is needed to understand the matter completely.

APPENDIX. EXAMPLeS FOR $f^{\eta}(Y)=f_{\Theta} \eta(Y)$ with $Y=q^{-n s}$

1. $f^{(1, n-1)}(Y)=1+\left[q^{n-1}+\cdots+q^{2}+q\right] Y$.

2. $f^{(1,3)}(Y)=1+\left[q^{3}+q^{2}+q\right] Y$.

3. $f^{(2,2)}(Y)=1+\left[q^{3}+2 q^{2}+q\right] Y+\left[q^{4}\right] Y^{2}$.

4. $f^{(1,1,2)}(Y)=1+\left[2 q^{3}+3 q^{2}+2 q\right] Y+\left[q^{5}+2 q^{4}+q^{3}\right] Y^{2}$.

5. $f^{(1,1,1,1)}(Y)=1+\left[3 q^{3}+5 q^{2}+3 q\right] Y+\left[3 q^{5}+5 q^{4}+3 q^{3}\right] Y^{2}+\left[q^{6}\right] Y^{3}$.

6. $f^{(1,4)}(Y)=1+\left[q^{4}+q^{3}+q^{2}+q\right] Y$.

7. $f^{(2,3)}(Y)=1+\left[q^{4}+2 q^{3}+2 q^{2}+q\right] Y+\left[q^{6}+q^{5}+q^{4}\right] Y^{2}$.

8. $f^{(1,1,3)}(Y)=1+\left[2 q^{4}+3 q^{3}+3 q^{2}+2 q\right] Y+\left[q^{7}+2 q^{6}+3 q^{5}+2 q^{4}+q^{3}\right] Y^{2}$.

9. $f^{(1,2,2)}(Y)=1+\left[2 q^{4}+4 q^{3}+4 q^{2}+2 q\right] Y+\left[q^{7}+4 q^{6}+5 q^{5}+4 q^{4}+\right.$ $\left.q^{3}\right] Y^{2}+\left[q^{8}+q^{7}\right] Y^{3}$.

10. $f^{(1,1,1,2)}(Y)=1+\left[3 q^{4}+6 q^{3}+6 q^{2}+3 q\right] Y+\left[3 q^{7}+8 q^{6}+11 q^{5}+8 q^{4}+\right.$ $\left.3 q^{3}\right] Y^{2}+\left[q^{9}+3 q^{8}+3 q^{7}+q^{6}\right] Y^{3}$.

11. $f^{(1,1,1,1,1)}(Y)=1+\left[4 q^{4}+9 q^{3}+9 q^{2}+4 q\right] Y+\left[6 q^{7}+16 q^{6}+22 q^{5}+\right.$ $\left.16 q^{4}+6 q^{3}\right] Y^{2}+\left[4 q^{9}+9 q^{8}+9 q^{7}+4 q^{6}\right] Y^{3}+\left[q^{10}\right] Y^{4}$. 
12. $f^{(2,2,2,2)}(Y)=1+\left[3 q^{7}+9 q^{6}+15 q^{5}+18 q^{4}+15 q^{3}+9 q^{2}+3 q\right] Y+\left[3 q^{13}+\right.$ $17 q^{12}+41 q^{11}+77 q^{10}+104 q^{9}+119 q^{8}+104 q^{7}+77 q^{6}+41 q^{5}+17 q^{4}+$ $\left.3 q^{3}\right] Y^{2}+\left[q^{18}+12 q^{17}+39 q^{16}+86 q^{15}+145 q^{14}+194 q^{13}+214 q^{12}+\right.$ $\left.194 q^{11}+145 q^{10}+86 q^{9}+39 q^{8}+12 q^{7}+q^{6}\right] Y^{3}+\left[3 q^{21}+17 q^{20}+41 q^{19}+\right.$ $\left.77 q^{18}+104 q^{17}+119 q^{16}+104 q^{15}+77 q^{14}+41 q^{13}+17 q^{12}+3 q^{11}\right] Y^{4}+$ $\left[3 q^{23}+9 q^{22}+15 q^{21}+18 q^{20}+15 q^{19}+9 q^{18}+3 q^{17}\right] Y^{5}+\left[q^{24}\right] Y^{6}$.

13. $f^{(1,2,2,2)}(Y)=1+\left[3 q^{6}+8 q^{5}+12 q^{4}+12 q^{3}+8 q^{2}+3 q\right] Y+\left[3 q^{11}+\right.$ $\left.14 q^{10}+30 q^{9}+48 q^{8}+54 q^{7}+48 q^{6}+30 q^{5}+14 q^{4}+3 q^{3}\right] Y^{2}+\left[q^{15}+9 q^{14}+\right.$ $\left.24 q^{13}+44 q^{12}+58 q^{11}+58 q^{10}+44 q^{9}+24 q^{8}+9 q^{7}+q^{6}\right] Y^{3}+\left[2 q^{17}+\right.$ $\left.8 q^{16}+14 q^{15}+17 q^{14}+14 q^{13}+8 q^{12}+2 q^{11}\right] Y^{4}+\left[q^{18}+q^{17}\right] Y^{5}$.

14. $f^{(1,2,3,4)}(Y)=1+\left[3 q^{9}+8 q^{8}+14 q^{7}+19 q^{6}+21 q^{5}+19 q^{4}+14 q^{3}+\right.$ $\left.8 q^{2}+3 q\right] Y+\left[3 q^{17}+14 q^{16}+36 q^{15}+73 q^{14}+118 q^{13}+166 q^{12}+200 q^{11}+\right.$ $\left.215 q^{10}+200 q^{9}+166 q^{8}+118 q^{7}+73 q^{6}+36 q^{5}+14 q^{4}+3 q^{3}\right] Y^{2}+\left[q^{24}+\right.$ $9 q^{23}+30 q^{22}+77 q^{21}+154 q^{20}+264 q^{19}+392 q^{18}+516 q^{17}+605 q^{16}+$ $639 q^{15}+605 q^{14}+516 q^{13}+392 q^{12}+264 q^{11}+154 q^{10}+77 q^{9}+30 q^{8}+$ $\left.9 q^{7}+q^{6}\right] Y^{3}+\left[2 q^{29}+10 q^{28}+33 q^{27}+80 q^{26}+158 q^{25}+268 q^{24}+395 q^{23}+\right.$ $518 q^{22}+606 q^{21}+640 q^{20}+606 q^{19}+518 q^{18}+395 q^{17}+268 q^{16}+158 q^{15}+$ $\left.80 q^{14}+33 q^{13}+10 q^{12}+2 q^{11}\right] Y^{4}+\left[q^{33}+5 q^{32}+17 q^{31}+39 q^{30}+75 q^{29}+\right.$ $119 q^{28}+164 q^{27}+197 q^{26}+210 q^{25}+197 q^{24}+164 q^{23}+119 q^{22}+75 q^{21}+$ $\left.39 q^{20}+17 q^{19}+5 q^{18}+q^{17}\right] Y^{5}+\left[q^{35}+3 q^{34}+7 q^{33}+12 q^{32}+16 q^{31}+\right.$ $\left.18 q^{30}+16 q^{29}+12 q^{28}+7 q^{27}+3 q^{26}+q^{25}\right] Y^{6}$.

15. $f^{(1,1,1,1,1,1)}(Y)=1+\left[5 q^{5}+14 q^{4}+19 q^{3}+14 q^{2}+5 q\right] Y+\left[10 q^{9}+35 q^{8}+\right.$ $\left.66 q^{7}+80 q^{6}+66 q^{5}+35 q^{4}+10 q^{3}\right] Y^{2}+\left[10 q^{12}+35 q^{11}+66 q^{10}+80 q^{9}+\right.$ $\left.66 q^{8}+35 q^{7}+10 q^{6}\right] Y^{3}+\left[5 q^{14}+14 q^{13}+19 q^{12}+14 q^{11}+5 q^{10}\right] Y^{4}+\left[q^{15}\right] Y^{5}$. 16. $f^{(1,1,1,1,2,2,2)}(Y)=1+\left[6 q^{9}+23 q^{8}+52 q^{7}+82 q^{6}+95 q^{5}+82 q^{4}+52 q^{3}+\right.$ $\left.23 q^{2}+6 q\right] Y+\left[15 q^{17}+82 q^{16}+257 q^{15}+518 q^{14}+1033 q^{13}+1519 q^{12}+\right.$ $1894 q^{11}+2037 q^{10}+1894 q^{9}+1519 q^{8}+1033 q^{7}+518 q^{6}+257 q^{5}+82 q^{4}+$ $\left.15 q^{3}\right] Y^{2}+\left[20 q^{24}+135 q^{23}+492 q^{22}+1295 q^{21}+2711 q^{20}+4757 q^{19}+\right.$ $7206 q^{18}+9597 q^{17}+11355 q^{16}+12003 q^{15}+11355 q^{14}+9597 q^{13}+7206 q^{12}+$ $\left.4757 q^{11}+2711 q^{10}+1295 q^{9}+492 q^{8}+135 q^{7}+20 q^{6}\right] Y^{3}+\left[15 q^{30}+124 q^{29}+\right.$ $509 q^{28}+1469 q^{27}+3344 q^{26}+6373 q^{25}+10495 q^{24}+15233 q^{23}+19730 q^{22}+$ $22978 q^{21}+24165 q^{20}+22978 q^{19}+19730 q^{18}+15233 q^{17}+10495 q^{16}+$ $\left.6373 q^{15}+3344 q^{14}+1469 q^{13}+509 q^{12}+124 q^{11}+15 q^{10}\right] Y^{4}+\left[6 q^{35}+\right.$ $65 q^{34}+304 q^{33}+946 q^{32}+2264 q^{31}+4463 q^{30}+7524 q^{29}+11099 q^{28}+$ $14529 q^{27}+17025 q^{26}+17939 q^{25}+17025 q^{24}+14529 q^{23}+11099 q^{22}+$ $\left.7524 q^{21}+4463 q^{20}+2264 q^{19}+946 q^{18}+304 q^{17}+65 q^{16}+6 q^{15}\right] Y^{5}+$ $\left[q^{39}+18 q^{38}+102 q^{37}+342 q^{36}+837 q^{35}+1628 q^{34}+2641 q^{33}+3672 q^{32}+\right.$ $4449 q^{31}+4739 q^{30}+4449 q^{29}+3672 q^{28}+2641 q^{27}+1628 q^{26}+837 q^{25}+$ $\left.342 q^{24}+102 q^{23}+18 q^{22}+q^{21}\right] Y^{6}+\left[2 q^{41}+17 q^{40}+62 q^{39}+145 q^{38}+\right.$ $250 q^{37}+341 q^{36}+377 q^{35}+341 q^{34}+250 q^{33}+145 q^{32}+62 q^{31}+17 q^{30}+$ $\left.2 q^{29}\right] Y^{7}+\left[q^{42}+4 q^{41}+6 q^{40}+4 q^{39}+q^{38}\right] Y^{8}$. 


\section{ACKNOWLEDGMENT}

The author is grateful to the referee of a previous version of this paper for suggesting the identity in $\S 4$.

\section{REFERENCES}

1. C. J. Bushnell and I. Reiner, Zeta functions of arithmetic orders and Solomon's conjectures, Math. Z. 173 (1980), 135-161.

2. _ L L-functions of arithinetic orders and asymptotic distribution of ideals, J. Reine Angew. Math. 327 (1981), 156-183.

3. _ Functional equations for L-series of arithmetic orders, J. Reine Angew. Math. 329 (1981), 88-124.

4. __ Zeta functions of hereditary orders and integral group rings, Texas Tech. Univ. Math Ser. 14 (1980), 71-94.

5. _ A survey of analytic methods in noncommutative number theory, in Orders and Their Applications (I. Reiner and K. W. Roggenkamp, eds.), Proc. 1984 Lecture Notes in Math., vol. 1142, Springer-Verlag, Berlin and New York, 1985, pp. 50-86.

6. L. Carlitz, q-Bernoulli and Eulerian numbers, Trans. Amer. Math. Soc. 76 (1954), 332-350.

7. M. Denert and J. Van Geel, Cancellation property for orders in non Eichler division algebras over global function fields, J. Reine Angew. Math. 368 (1986), 165-171.

8. _. The class number of hereditary orders in non-Eichler $(R)$-algebras over global function fields, Math. Ann. 282 (1988).

9. M. Denert, Affine and projective orders in central simple algebras over global function fields (An analytic approach to the ideal theory), Thesis, State University of Ghent, 1987.

10. __ Solomon's second conjecture: a proof for local hereditary' orders in central simple algebras, preprint.

11. I. Reiner, Maximal orders, Academic Press, New York, 1975.

12. L. Solomon, Zeta functions and integral representation theory, Adv. in Math. 26 (1977), 306326.

13. A. Weil, Adeles and algebraic groups, Institute for Advanced Studies, Princeton, N.J., 1961.

Seminar for Algebra and Functional Analysis, State University of Ghent, Galglaan 2, 9000 GHeNt, BeLgIUM 